\title{
Longitudinal Incision Versus Transverse Incision in Trigger Finger Release Surgery
}

W.A.Kandel, O.M.Essawy and M.G.Z.Botrous

Orthopedic Surgery Dept., Faculty of Medicine, Benha Univ., Benha, Egypt

E-Mail:dr_Michael@yahoo.com

\begin{abstract}
Setting off of a flexor ligament, otherwise called stenosing synovitis, is a typical hand pathology as often as possible seen and treated by muscular specialists. The point of this forthcoming investigation was to look at longitudinal versus cross over cuts in trigger finger discharge as respect utilitarian result, patients fulfillment and specialist inclination. This planned randomized investigation on twenty fingers went through careful delivery by means of a longitudinal cut, and the other twenty fingers by means of a cross over cut. Patients were assessed in the outpatient setting at 2, 8, and 36 weeks by their treating specialist. At each subsequent visit, both the patient and treating doctor finished the PSAS and OSAS, separately (alluded to in mix as the POSAS; Version 2. The DASH was likewise gathered at the 8-and 40-weeks postoperative.there were no huge contrasts in scar quality measurements among cross over and longitudinal entry points. Mean PSAS scores at about two months were 15.9 SD 2.2 and 15.6 SD 2.0 for cross over and longitudinal gatherings, separately. There were no critical contrasts in the DASH scores among cross over and longitudinal cut gatherings preoperatively. The DASH scores improved from a mean of 29 preoperatively to 17 at about two months $(p<0.05)$, and to 4 and no more ongoing development $(\mathrm{p}<0.05)$. Despite the fact that there was no factually huge distinction between the result of the patients with cross over versus longitudinal entry points in regards to the usable time, practical improvement, and patient fulfillment, there was more specialist inclination with the longitudinal cut as it permits better openness to A1 pulley, conveys less danger of neurovascular injury, and permits early scope of finger movement.
\end{abstract}

\section{Introduction}

Setting off of a flexor ligament, otherwise called stenosing synovitis, is a typical hand pathology every now and again observed and treated by muscular specialists. It was first depicted by Alphonse Notta in 1850 , the excruciating clicking while at the same time flexing/broadening the finger was the explanation of the name (Trigger). Trigger finger is an impingement at the degree of the hypertrophic first annular (A1) pulley because of an irritation determined size inconsistency of the elaborate flexor tendons [1]. When moderate treatment, (for example, bracing or corticosteroid infusion) fizzled, careful delivery was indicated [2].

The site and state of the cut with respect to hand work and anatomic contemplations are significant for the achievement of the medical procedure. There is diverse skin cuts depicted to deliver the A1 pulley. Which cut the specialist will pick doubtlessly relies upon the careful preparing pick up from his careful seniors as opposed to having encountered and tried numerous cuts himself [3].

Probably, the present condition has prompted solid feelings among certain specialists about which entry point method is better over another [2]. Although trigger finger discharge is considered as one of the littlest elective hand a medical procedure technique, most hand specialists found that there is a lot of patients that will give a drawn out recuperation period normally because of scar development alongside ensuing impediments in day by day exercises [4].

The ligaments that flex the fingers coast effectively with the assistance of pulleys which hold the ligaments near the bone. This is like how a line is hung on a casting pole. Trigger finger happens when the pulley turns out to be too thick, so the ligament can't coast effectively through it [5].

Early suggestions was careful treatment of trigger finger as it was clear and profoundly compelling, while delayed moderate treatment was temperamental and expensive [6]. Local infusion is currently commonly consented to be the main line of treatment. Medical procedure is saved for people in whom infusion therapy has fizzled or because of other pathology [7].

In trigger finger discharge a medical procedure, both cross over and longitudinal cuts are normally utilized. For our situation arrangement, careful arrival of the trigger fingers had a palatable result, with early recuperation of the patients, and with no genuine difficulties. There was no factually critical distinction between patients with cross over and longitudinal cuts in regards to employable time, useful improvement, and patient fulfillment. This may be because of that the longitudinal cut may permit more satisfactory introduction and simple availability to longitudinally arranged A1 pulley [8].

Additionally, the longitudinal cut may convey less danger of injury to the hidden neurovascular groups as it was made between the nerves, so it was by and large a more secure way to deal with the ligament sheath. Albeit all instances of the two gatherings recaptured a full ROM of MP joint of the worked finger at last development, ROM could be permitted from the get-go in patients with longitudinal cut as it was in line of finger range, and makes less twisted pressure and a more modest scar, rather than patients with the cross over cut that the line of finger movement make the injury in tension [9].

The point of this planned investigation was to analyze longitudinal versus cross over cuts in trigger finger discharge as respect useful result, patients fulfillment and specialist inclination.

\section{Patient and method}

This prospective randomized controlled trial was approved by the Research Ethics Committee of the 
Benha University. Informed consent was obtained from each patient.

Patients with a previous failed surgical A1 pulley release and those with trigger thumb were excluded. A shared decision-making model was used to counsel patients about non-operative and operative treatment options. Those electing to proceed with open surgical release of the A1 pulley were offered the option to participate in the study.

Twenty fingers underwent surgical release via a longitudinal incision, and the other twenty fingers via a transverse incision. A full detailed history was obtained regarding duration of symptoms and the previous management, the effect of pain on the level of activity, and the time until presentation.

\section{Inclusion criteria \\ - Males and Females \\ - Age group from 30 to 60 years old . \\ - All fingers. \\ - Any medical co morbidity.(e.g. Diabetes,Rheumatoid.....)}

- Failure of conservative treatment for at least 3 months or after at least one local injection of corticosteroids.

- Multiple fingers affected or association with carpal tunnel syndrome

\section{Exclusion criteria}

- Recurrent cases were excluded from this study.

Enrolled patients were randomized in equal numbers to undergo trigger finger release through either a transverse or longitudinal incision using a computergenerated sequence in a random permuted block fashion using Microsoft Excel, in blocks of two or four, with random variation of the block size. This made it impossible to predict which incision group the next patient would be assigned to. A stack of opaque envelopes was prepared to implement the randomization, each containing a slip of paper listing the primary incision type. The envelope was opened in the sequence written on the outside of the envelope, being opened in the preoperative holding area on the day of surgery, and only after the patient was first officially enrolled into the study. This guarded against incision-type switching, since the sequence and group assignment were known to be continually audited.

Trigger finger discharge was performed under neighborhood sedative with or without sedation, in view of patient inclination. Tourniquet utilize was needy upon specialist's inclination. Cross over entry points were made along the proximal palmar wrinkle for the pointer, along the distal palmar wrinkle for the ring and little fingers, and in-accordance with the proximal and distal palmar wrinkles for the long finger. Longitudinal cuts were made straightforwardly over the midline of the influenced digit, with the proximal degree situated at the proximal palmar wrinkle for the pointer, at the distal palmar wrinkle for the ring and little fingers, and inaccordance with the proximal and distal palmar wrinkles for the long finger - these were focused over the substantial knobs.

The Clinical evaluation was resolved by green's characterization. Straightforwardly after medical procedure, all patients were told to utilize the influenced hand with no particular restrictions. Fourteen days postoperatively, the injury was assessed again and the stitches were eliminated.

Patients were assessed in the outpatient setting at 2, 8 , and 36 weeks by their treating specialist. Fourteen days postoperatively patients were likewise assessed and treated by a confirmed hand specialist.

At each subsequent visit, both the patient and treating doctor finished the PSAS and OSAS, separately (alluded to in blend as the POSAS; Version 2).

The DASH was likewise gathered at the 8-and 40weeks postoperative visits. Difficulties (intermittent/tenacious manifestations, wound dehiscence, wound seepage, need for anti-toxins or nearby twisted consideration, contamination, ligament bowstringing, flexion constriction, or extra medical procedure) were recorded at each development.

\section{Results}

The randomization process, with $71 \%$ (61/86) of patients available for follow-up at a mean of 8 weeks, and $60 \%$ at a mean of 40 weeks postoperatively. Patient baseline characteristics, and statistical comparisons between groups, are provided in Table (1).

At 8 and 54 weeks postoperative, there were no significant differences in scar quality metrics between transverse and longitudinal incisions. Mean PSAS scores at 8 weeks were 15.9 SD 2.2 and 15.6 SD 2.0 for transverse and longitudinal groups, respectively. PSAS scores at 54 weeks were 10.7 SD 1.7 and 9.3 SD 1.0, OSAS scores at 8 weeks were 14.6 SD 2.0 and 12.5 SD 1.1 , and OSAS scores at 54 weeks were $9.0 \mathrm{SD} 1.1$ and 8.1 SD 0.5.

Among patients receiving both incision types for multiple simultaneous trigger releases, there were no significant differences in PSAS or OSAS scores at either time-point between incision types (Table 2). Scoring the overall opinion of both scar types, as compared with normal skin, was similar between transverse and longitudinal incisions when evaluated by patients and observers/physicians.

There were no significant differences in the DASH scores between transverse and longitudinal incision groups preoperatively. The DASH scores improved from a mean of 29 preoperatively to 17 at 8 weeks $(\mathrm{p}<0.05)$, and to 4 at most recent follow-up ( $p<0.05)$.

There were no differences between transverse or longitudinal incision types at any time-point. No differences in DASH score improvement were observed between 0 and 8 weeks $(p=0.85)$, or between 0 and 54 weeks $(\mathrm{p}=0.14)$, when comparing incision types.

The mean DASH Score preoperative was $47.63 \pm 8.81$ points (ranging from 34.1 to 63.6). According to the DASH score, all patients have preoperative poor hand function (score $>30$ ). 
The mean DASH scores in transverse incision group had significantly decreased by 6.5 points 1 month postoperatively, to become 27.4 points 3 months postoperative and reached15.6 points at the final follow up $(\mathrm{p}<0.01)$.

Longitudinal incision group had significantly decreased by 12 points 1 month postoperatively, to become 19.2 points 3 months postoperative and reached 9 points at the final follow up $(\mathrm{p}<0.01)$. There were no statistically significant difference in improvement of mean DASH scores between the two groups $(p=0.87)$

Almost all wounds healed and sutures removed within 15 days (range 12-21 days post- operative). All cases of both groups regained a full ROM of MP joint of the operated finger at the final follow up.

Table (1) Patient information.
Regarding patients' satisfaction after doing the surgery, 38 patients were completely satisfied, 2 patients were satisfied with some reservations. These two patients were related to longitudinal incision group, and had a superficial infection in the form of local redness and hotness without discharge and were treated by systemic antibiotics and regular dressing for about 3 weeks post operative.

The mean operative time from skin incision to closure was 9 minutes for transverse incision (range 7-14 minutes) while for longitudinal incision was 6 minutes (range 4-10 minutes). Although the surgical release using longitudinal incision was shorter in duration than that of transverse incision, there was no statistically significant difference in the operative time between the two groups $(\mathrm{p}=0.65)$.

\begin{tabular}{|c|c|c|c|c|c|c|c|}
\hline \multirow[b]{2}{*}{ Demographic factor } & \multicolumn{4}{|c|}{ With longitudinal } & \multicolumn{2}{|c|}{ With transverse } & \multirow[b]{2}{*}{ p-value $^{a}$} \\
\hline & All & atients & & ision & & ision & \\
\hline Number of patients & 40 & & 20 & & 20 & & N/A \\
\hline Number of unique incisions & 40 & & 20 & & 20 & & N/A \\
\hline Age (years; mean SD) & 61 & SD 11 & 61 & SD 12 & 61 & SD 10 & 0.88 \\
\hline Diabetes & 24 & $(39 \%)$ & 13 & $(39 \%)$ & 11 & $(39 \%)$ & $>0.99$ \\
\hline Multiple simultaneous releases & 19 & $(31 \%)$ & 9 & $(27 \%)$ & 10 & $(36 \%)$ & 0.58 \\
\hline Prior steroid injection & 44 & $(72 \%)$ & 23 & $(70 \%)$ & 21 & $(75 \%)$ & 0.78 \\
\hline Sex (female) & 25 & $(41 \%)$ & 12 & $(36 \%)$ & 13 & $(46 \%)$ & 0.44 \\
\hline Smoking & 2 & $(3 \%)$ & 0 & $(0 \%)$ & 2 & $(7 \%)$ & 0.21 \\
\hline Veterans administration status & 19 & $(31 \%)$ & 10 & $(30 \%)$ & 9 & $(32 \%)$ & $>0.99$ \\
\hline
\end{tabular}

Table (2) Patient and PSAS and OSAS scores among patients with both incision types for multiple simultaneous trigger finger releases.

\begin{tabular}{lccc}
\hline & $\begin{array}{c}\text { Transverse } \\
\text { incision }\end{array}$ & $\begin{array}{c}\text { Longitudinal } \\
\text { incision }\end{array}$ & p-value \\
\hline Patient Scar Assessment Scale (PSAS) score & & & 0.66 \\
8 weeks follow-up $(\mathbf{n}=\mathbf{1 1})$ & 19.8 & 18.9 & 0.10 \\
54 weeks follow-up $(\mathbf{n}=\mathbf{1 2})$ & 14.7 & 10.1 & 0.53 \\
Observer Scar Assessment Scale (OSAS) score & 13.8 & 14.6 & 0.24 \\
8 weeks follow-up $(\mathbf{n}=\mathbf{1 1})$ & 10.3 & 8.6 & \\
54 weeks follow-up $(\mathbf{n}=\mathbf{1 2})$ & & & \\
\hline
\end{tabular}

\section{Discussion}

Among patients getting both cut sorts for synchronous arrivals of various trigger digits, we didn't notice a distinction in PSAS or OSAS scores between cut kinds. We neglected to dismiss our essential invalid speculation and reason that cross over and longitudinal cut sorts both yield correspondingly satisfactory scar quality after trigger finger discharge.

The optional goal of this examination was to assess whether cut sort influenced quiet announced results following trigger finger discharge, as stop mined utilizing the DASH. Additionally, the greatness in progress in the DASH scores somewhere in the range of 0 and two months, and somewhere in the range of 0 and 54 weeks, was the same between gatherings. We neglected to dismiss our auxiliary invalid speculation and infer that dependable improvement in patient-revealed handicap happens following trigger finger discharge, whether or not cross over or longitudinal entry point types are utilized.

Our examination lines up with past investigations, which show in general fantastic results and negligible inconveniences after open treatment for trigger finger [10].

Our noticed improvement in the DASH from 29 preoperatively to 4 at 54 weeks post-operatively recommends that trigger delivery patients experience negligible furthest point incapacity following recuperation. This is steady with earlier investigations detailing a careful achievement rate moving toward $100 \%$ for trigger digit discharge [11]. Our complexity rate was like the $1-13 \%$ rate revealed in earlier arrangement [12].

There was study [13], which is distributed an imminent, randomized examination contrasting quantitative scar volume and clinical out-separates three 
entry point types for trigger finger discharge acted in 32 patients. They assessed longitudinal, distally based cross over, and proximally based cross over entry point types. They noticed no huge contrast between scar volumes, as dictated by ultrasound imaging, between the three gatherings at 12 weeks postoperatively. While quantitative scar volume may fill in as a proxy of scar quality, no approved strategies were utilized to assess scars.

There were no huge contrasts in the DASH scores between the three gatherings at 1, 3, and a year postoperatively; be that as it may, the creators suggested utilization of longitudinal cuts dependent on noticed huge improvement in the DASH score by multi month postoperatively.

Further, the creators exhorted against distally based cross over entry points in the distal palmar wrinkle because of their noticed non-critical expansion in scar volume and specialized trouble of everting the skin edges during conclusion. That review was restricted by a little example size and absence of intensity counts. Moreover, patients from the longitudinal cut gathering detailed altogether more prominent standard dis-capacity on the DASH than the other two entry point gatherings, in spite of randomization. Our investigation develops the discoveries of Kloeters et al. (2016) through utilization of approved scar evaluation result instruments (Singer et al., 2007), utilization of intensity computations, and exhibit of comparative gauge attributes following randomization.

Our examination has a few impediments. Despite the fact that this was a forthcoming randomized preliminary, we were unable to dazzle the patient or specialist to the entry point type. It is hazy whether the $29 \%$ of patients lost to development would change the discoveries of the examination whenever included. Force computations were played out from the earlier to decide suitable example sizes; nonetheless, we couldn't put together these with respect to insignificant clinically significant contrast information, which is inadequate in the writing for the POSAS measurements.

The set number of confusions blocked important factual examination among gatherings; and given the general low difficulty pace of trigger finger discharge, we expected that the investigation would require an unnecessary number of patients to enlist to accomplish suitable force for this optional result.

In spite of the fact that we recorded the DASH scores and presence of proximal interphalangeal joint flexion contractures, we didn't officially quantify scope of movement and can't remark with respect to contrasts between bunches in such manner. We recorded the quantity of patients requested to take on the investigation, anyway it is conceivable that not all refusals were logged - it is hazy whether this would influence our outcomes. For patients going through arrival of more than one digit, we decided to randomize the principal digit and dole out the leftover digits the other entry point type. It is hazy if our outcomes would vary had we randomized every individual entry point all things being equal.

Other cut sorts, and varieties thereof, have been accounted for trigger finger discharge. By assessing just cross over and longitudinal entry points, the generalizability of our discoveries might be restricted.

For our situation arrangement, careful arrival of the trigger fingers had an exceptionally acceptable result, with early recuperation of the patients, and with no genuine difficulties.

There was no measurably critical distinction between patients with cross over and longitudinal cuts in regards to employable time, utilitarian improvement, and patient fulfillment.

Nonetheless, all the ten specialists' agreement that cases with longitudinal cut had more limited mean employable time. This may be because of that the longitudinal cut may permit more sufficient presentation and simple availability to longitudinally arranged A1 pulley [7].

Likewise, the longitudinal entry point may convey less danger of injury to the fundamental neurovascular packs as it was made between the nerves, so it was for the most part a more secure way to deal with the ligament sheath.

Albeit all instances of the two gatherings recaptured a full ROM of MP joint of the worked finger at last development, ROM could be permitted from the get-go in patients with longitudinal entry point as it was in line of finger range, and makes less twisted pressure and a more modest scar, rather than patients with the cross over cut that the line of finger movement make the injury in strain.

Antagonistic occasions somewhere in the range of 5 and $36 \%$ in the trigger finger discharge have been accounted for, including repetitive setting off, nerve injury and wound mending issues, for example, contaminations, wound bonds, and agonizing scar tissue disturbance. The most revealed grumblings were wound mending disturbances just as torment and delicacy of scar tissue [8].

In our investigation, shallow contamination had happened in two cases with longitudinal cut gathering though no shallow disease had happened with cross over cut gathering. In any case, this complexity may be identified with understanding general condition, the sanitization technique, or inadequate injury care, however unquestionably was not identified with the kind of entry point.

In the uncommon occasion of a disease that requires careful seepage, it's really simple to stretch out the longitudinal entry point to clean out the contamination. This additional more incentive to the utilization of that cut.

\section{Conclusion}

Although there was no statistically significant difference between the outcome of the patients with transverse versus longitudinal incisions regarding the operative time, functional improvement, and patient 
satisfaction, there was more surgeon preference with the longitudinal incision as it allows better accessibility to A1 pulley, carries less risk of neurovascular injury, and allows early range of finger motion.

\section{References}

[1] S.Ahktar, M.J.Bradley, D.N.Quinton. Clinical review: management and referral for trigger finger/thumb. BMJ, Vol.331, PP.3033.331.7507.30,2005.

[2] A.H.Makkouk, M.E.Oetgen, C.R.Swigart. Trigger finger: etiology, evaluation, and treatment. Curr Rev Musculoskelet Med, Vol.1(2), PP.92-96,007-90121,2008 .

[3] Jia.Wang, Jia-Guo Zhao, Cong-Cong Liang. "Percutaneous release, open surgery, or corticosteroid injection, which is the best treatment method for trigger digits?." Clinical Orthopaedics and Related Research® 471.6 (2013): 1879-1886.Unglaub F, Cakmak F, Wolf MB, Hahn P. Letter regarding "Adverse events of open A1 pulley release for idiopathic trigger finger" J Hand Surg, Vol.37(11), PP.2428-2429. doi: 10.1016/j.jhsa.2012.08.038,2012.

[4] P.L.Hudak, P.C.Amadio, C.Bombardier. Development of an upper extremity outcome measure: the DASH (disabilities of the arm, shoulder and hand) [corrected]. The upper extremity collaborative group (UECG) Am J Ind Med, Vol.29(6), PP.602-608. doi: 10.1002/(SICI)10970274(199606)29:63.0.CO;2-L,1996.

[5] C.E.Rhoades, R.H.Gelberman, J.F.Manjarris. Stenosing tenosynovitis of the fingers and thumb. Results of a prospective trial of steroid injection and splinting. ClinOrthopRelat Res, Vol. Nov, PP. 2368,1984 .

[6] B.M.Huisstede, P.Hoogvliet, J.H.Coert. European HANDGUIDE Group. Multidisciplinary consensus guideline for managing trigger finger: results from the European HANDGUIDE Study. PhysTher, Vol. Oct. 94 (10), PP.1421-33,2014.

[7]

[8] Green David. Operative Hand Surgery. 2nd edn. Churchill Livington, Vol.55, PP. 2124,1988.

[9] H.Tsuchie, T.Nishi, H.Abe. Trigger finger appearing as gradually increasing digital nerve disorder after surgical treatment. Case Rep Orthop, Vol.013, PP.542965,2013.

[10] Tabl, Eslam Abdelshafi; Kandil, Mahmoud Ibrahim. Longitudinal Versus Transverse Incision In Trigger Finger Release. A Prospective Comparative Study. Al-Azhar Assiut Medical Journal, Vol. 13.3, PP. 2,2015.

[11] M.H.Lim, K.K.Lim, M.Z.Rasheed. Outcome of open trigger digit release. J Hand Surg Eur.vol, 32, PP.457-9,2007.

[12]R.J.Stefanich, C.A.Peimer,. Longitudinal incision for trigger finger release. J Hand Surg Am, Vol. 14, PP. 316-7,1989.

[13]W.T.Lee, A.K.Chong. Outcome study of open trigger digit release. J Hand Surg Eur, Vol. 36, PP.339,2011.

[14] O.Kloeters, D.J.Ulrich, G.Bloemsma. Comparison of three different incision techniques in a1 pulley release on scar tissue formation and postoperative rehabilitation. Arch Orthop Trauma Surg, Vol.136, PP. 731-7,2016. 